\title{
2. Platform Genealogies
}

\begin{abstract}
In Chapter 2, "Platform genealogies," we consider the continuities and discontinuities around LINE, WeChat, Facebook and WhatsApp as a digital genealogy. We explore the particular histories and practices informing those platforms - what Gillespie (2015) calls "the politics of platforms" or Lamarre calls (2017) "platformativity" — and why they are being adopted intergenerationally. For example, the developed and quick uptake of LINE was in direct response to the way in which mobile social media shifted in and after the earthquake, tsunami and Fukushima nuclear disaster of 2011 known as $3 / 11$.
\end{abstract}

Keywords: Platforms; LINE; WhatsApp; QQ; 3/11

In this book we explore the familial practices that shape and are shaped by social mobile media. Within different contexts-cultural, social, generational and familial - we can begin to de-naturalize the ways in which platforms create different affordances and practices. As we argue, through the negotiation of boundary work by families, mobile social media use and non-use is recalibrated. Rather than being platform-specific, this book considers some of the enduring practices and cultures that move in, across, and through the various platforms our participants used. As noted in Chapter 1, particular platforms dominant specific cultural contexts - for example, LINE in Japan, and WeChat in China.

The ways in which the affordances of platforms shape how people manage familial relations is significant - thus it is important to acknowledge that platforms come with their own interfaces that play a significant role in their successful uptake. Scholars such as Ian Bogost and Nick Montfort (2009) have been key in establishing the field of platform studies as an investigation of hardware and software design and the "creative works" produced on and by those systems. Their studies take into account the cultural dimensions

Hjorth, L., K. Ohashi, J. Sinanan, H. Horst, S. Pink, F. Kato, B. Zhou, Digital Media Practices in Households: Kinship through Data. Amsterdam: Amsterdam University Press, 2020 DOI 10.5117/9789462989504_CHO2 
of designed platforms, but these are often secondary to the technical and political economy dimensions.

As Tarleton Gillespie $(2010,359)$ observes in his study, discourses of specific platforms might "matter as much for what they hide as for what they reveal" He argues that these uses connote the concept-literal or metaphorical — of an expansive, level, nonhierarchical surface, hosting multiple activities or events. Platforms thus "anticipate" certain actions, implying a certain neutrality or egalitarian organization that provides an open or democratic space supporting users (individuals or corporations) (Ibid., 350). Such metaphors echo the rhetoric of Web 2.0 as a place facilitating user-created content, amateur creativity, production, creative practice and networking. Yet, as Gillespie demonstrates, the "comforting" ideas of free space and openness obfuscates the tensions and power relationships between the roles and actions of providers, users, and commercial media companies in the creation, distribution, and controlled delivery of online content. José van Dijck builds on Gillespie's discussion by arguing that we live in a "platform society" whereby platforms are "gateways" for everyday social life (2016). Understanding platforms as part of a genealogy means taking seriously the relationality of platforms and how they are part of a broader cultural, social and historical practice. As Hjorth et al. suggest:

We consider platforms as emerging as places in which the digital and material are entangled, and we account for how platforms are made through intensities of social engagement as much as through their occupation of physical sites. Platforms become central to thinking about the clustering of experiential and representational forms, as part of places that are made up of things, persons, and processes of different qualities and affordances, and as open, continually changing aspects of the environments we inhabit $(2016,31)$.

In this book we use the concept of genealogy as means of tracing of lines of descent, lines being an important concept for understanding the entanglement of bodies with the digital and material. For anthropologist Tim Ingold, "the entanglement of things has to be understood literally and precisely, not as a network of connections but as a meshwork of interwoven lines of growth and movement" (2010, n.p). Meshwork highlights the biographies, relations, and participation of objects, people and media.

Michel Foucault (2003) used the concept as a philosophical historical technique in which social beliefs are put into context. His work was particularly interested in power and the different way knowledges come into 
existence. Framing platforms in terms of genealogy allow for continuities and discontinuities, in which platforms as seen as transitory in the digital kinship lines of descent. Here we view platforms as biographies for practices. Given the significance of lines in many of the definitions of genealogy, this chapter will begin with one of the key platforms used in Japan, LINE. We then discuss WeChat in Shanghai and then Facebook, WhatsApp and Instagram in Melbourne. We conclude by discussing one of the enduring continuities across the three sites and multiple platforms-especially in terms of paralinguistics such as emojis ("picture characters"), emoticons (typographic characters), stamps and stickers.

\section{Japan: LINE: A Post 3/11 Social Media}

The social mobile media app LINE has become popular in Japan due to the particular technological, cultural, social, and linguistic factors informing the uptake and adoption of social and mobile media. As mentioned in the introduction, the success of LINE emerged in direct response to the way in which mobile social media shifted in and after the disaster of $2011(3 / 11)$ - as a result of declining trust in traditional media such as NHK (Hjorth and Kim 2011; Slater et al. 2012; ITmedia Business Online 2013; Steinberg 2019). The ex-president of LINE, Mr. Morikawa, noted that they decided to develop LINE because they saw many people use Twitter as a communication tool between intimates at the time of 3/11 (ITmedia Business Online 2013). LINE quickly became embedded in everyday social media practices.

Post 3/11 social mobile media-especially LINE — became integral to many people's everyday life and interpersonal relationships, especially for family communication. Some have suggested that LINE is the form of intergenerational communication for Japanese by enveloping mundane intimacies with hybrid forms of new media literacy. The digital genealogies of LINE reflect offline familial intimacies that both continue older familial rituals while also providing new ways for care at a distance, accounting for what Mizuko Ito and Daisuke Okabe (2005) have called intimate ambient co-presence. The adoption of LINE plays into broader historical practices of mobility in Japan that include long commutes as part of everyday life (Fujimoto 2005).

For Kenichi Fujimoto, understanding mobile media in Japan requires a differentiation between mobility and mobilism. Fujimoto defines mobilism as "broader cultural and social dimensions such as malleability, fluctuation and mobilization" $(2005,80)$. Unlike mobilism, "mobility has tended to refer to functional dimensions of portability and freedom from social and 
geographic constrain" (Ibid.). Thus, mobilism is tied to socio-geographic factors, whereas mobility infers transcendence, particularly around geographic constraints. The distinction between mobilism and mobility becomes important when considering mobile media as part of the fabric of cultural, social and geographic textures in everyday life.

In Japan we see how social mobile media enabled alternative ways to articulate the personal and intergenerational as well as the political and social. For example, immediately after $3 / 11$, reports suggest that in the central Tokyo area (including prefectures adjacent to Tokyo) more than five million people could not reach their home on that day because of the damage to public transportation. According to the survey conducted by Weather News (2014), it took about seven times longer to reach home when compared to one's ordinary commuting.

While many stayed in their office building, some people decided to walk home. During the walk home, many people tried to use Twitter to collect information about on-going changes in transportation recovery. One could, in fact, spontaneously change the route toward his/her home based on communication through tweets. Social media enabled coordination at a micro level. The experience of the earthquake motivated people to utilize social media applications to gather and share information about changes in the social and political climates (Slater et al. 2012; Gill, Steger and Slater 2013).

In addition, mobile media operated symbolically as a vessel for containing the intimate during times of grief - some people held onto their phones as security when they couldn't make contact with loved ones (Hjorth and Kim 2011). In particular, the rise of social mobile media like LINE in Japan highlights the significance of the media in maintaining and fostering intergenerational ties. These are personal as they are political; intimate, as they are public.

LINE remains an integral part of the social media landscape in Japan and its significance is marked by a cross-generational use, and in this way can be viewed as a metaphor for familial genealogies. LINE is a service providing text messaging among individuals or groups, as well as making phone and video calls for no charge. The Stamp function-an advanced form of paralinguistics such as emoji (pictogram or emoticons) and decomail-allows communication without the need of text messaging and the kawaii (cute) culture was key to its success. These functions allow for more affective care at a distance activity, providing co-present gestures of emotion and feeling that can be seen to transcend words and be more sensitive to social context than just words.

Byford (2013) notes that LINE in Japan has introduced the "next-level emoji" through their deployment of stickers (stamps). As we explore later, 


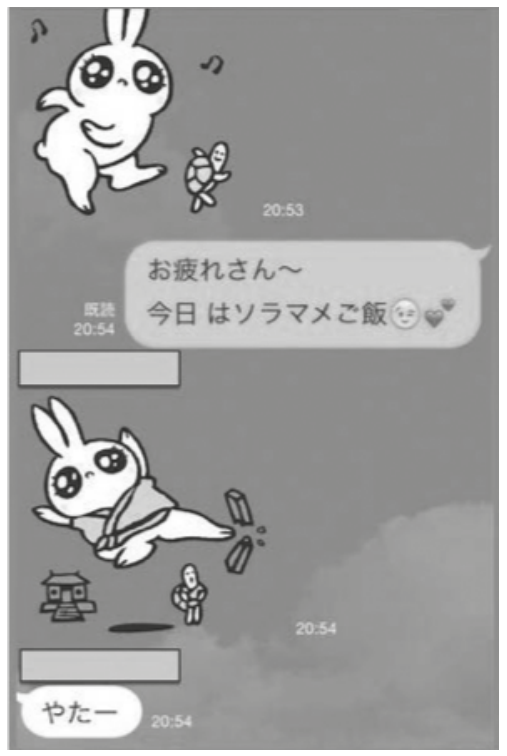

Figure 2.1: Chat with rabbit stamp on LINE

Stark and Crawford $(2015,1)$ define stickers as "proprietary to each platform that sells them." However, the ambiguities of emoji and stickers in and across work contexts like emails and social media can confuse and also be viewed as part of broader problematic of affective labor practices. In the case of families dealing with distance, they can provide the intimacy and care at a distance that is essential for maintaining intergenerational relationships.

In Japan, keitai cultures have afforded ways in which to express forms of intimacy previously left tacit (Ito, Matsuda and Okabe 2005). As noted previously, kawaii (cute) culture informed the invention of emojis in Japan in 1999. Kawaii cultures play with the premature adulthood and gender conventions (Kinsella 1995; Hjorth 2003a, 2003b, 2005) and play, affect and labor are entangled in particular ways. For example, the rise of mobile media (keitai) in Japan was marked by a personalization culture both inside and outside the device (Hjorth 2009). The rise of the online saw the further development of kawaii cultures through ASCII paralinguistics, especially by high school girls who creolized hiragana with ASCII in what has now been called the high school pager revolution (Fujimoto 2005; Ito et al. 2005; Hjorth 2009). Also, there is a long history of deploying the kawaii to make friendly or "warm" the coldest of new technologies (Hjorth 2003a).

By 2015, the number of domestic registrants for LINE was 58 million, or over $45 \%$ of the whole population (LINE 2015). At this time the popularity of LINE had overtaken the role of social media like mixi, GREE 
and Mobage. Deploying kawaii characters have played an important role in Japanese culture and expressing emotions (Hjorth 2003a, 2003b). LINE thus provided the growing amount of sumaho users with easy, fun, familiar, affective and mundane ways to communicate with copresent intimates. Here the kawaii functions to personalize and bring an emotional softening to digital encounters. The kawaii helps to embed particular feelings associated with the message, thus allowing for building emotional ties.

\section{China: WeChat}

On 21 January 2011, Tencent Technology officially launched its mobile instant messaging (IM) product WeChat, with the slogan "To connect people, to connect goods, and to connect everything." Tencent Technology understood the Chinese mobile and internet market well, having established QQ, one of the most important first-generation IM mobile apps. For many generations, QQ was their first entry into mobile internet — and for many of the older generations, it was synonymous with the internet.

WeChat has undergone six major iterations, evolving from one single IM product into one of the most popular mobile apps that combines communication, social networking, media and e-banking functions together. By June 2015, it reached a record 90\% coverage of mobile phone users. In the first quarter of 2015, the number of people engaging with WeChat reached 549 million, with many suggesting that the emergence of WeChat stickers (like LINE stamps) played a key role in this rapid uptake (Tencent 2016). WeChat had developed a very young user group base, with an average age of 26 , where $86.2 \%$ were young adults between the ages of 18 and 35 .

WeChat's friendly interface includes four major interactive componentsChats, Contacts, Discover and Me. As the basic function of WeChat, Chats is set as the default home display for WeChat users. In the Chats section, private chats (with single head portrait) and group chats (with multiple head portraits) are both available for users. Users can also subscribe to public accounts according to their interests.

Multiple sub-functions can be initiated within the Chats section. WeChat allows sending texts, photos, audio, videos, locations and one's favorites. The "Red Packet" and "Transfer" function allows money to be exchanged between WeChat users. The second most-used part of WeChat is the third icon on the bottom, "Discover." In the Discover section, users can find the social function of WeChat, i.e., "Moments." 
New feeds in WeChat Moments are displayed in the informational flow style - similar to Facebook in design. Users can choose to "check-in" when sharing a feed to Moments. In the right screenshot, information in the red rectangle is a snapshot of the "check-in." The way of being social in Moments is by selecting "like" or "comment" on each post. All posts of one single user are date stamped as his or her posts, including all text, image and online content posts. Locative information is also displayed in the individual post page. In the screenshot above, information in the red rectangle is another sample of "check-in." The fourth part is the personal function of WeChat, which includes supportive functions of WeChat, for example, Wallet (for WeChat Pay).

It took WeChat over five years to grow from a simple instant messaging app to a comprehensive social and e-commerce app. However, it is significant that the broad uptake of WeChat is not necessarily represented by youth but, rather, through the engagement with multi-generational functions. In China, it is now very fashionable for families to have a family WeChat group. Like LINE in Japan, WeChat in China can be seen as an extension of kinship rituals. A family WeChat group provides an online private place for domestic communication, and this is especially useful for families when they are physically apart, as is increasingly becoming the case. The easy use and almost zero cost of WeChat makes communication affordable for everyone; the media rich function of WeChat makes communication on WeChat more vivid and multi-dimensional. WeChat brings distant family members together and creates a sense of co-presence.

25-year-old Yolanda is a key example of someone who used WeChat to extend the lines of connection between her and her family when she moved away to pursue a degree in International Relations in Shanghai. Yolanda's hometown was far from Shanghai, and so she only traveled back home to visit her parents - who worked as civil servants - three times each year. For this reason, she established a WeChat family group, and the family moved in and out of the group throughout the day to discuss everyday events, feelings and family rituals. However, Yolanda's main focus was to keep in contact with her peers on WeChat, as they were all frequent users. For Yolanda, texting was not as flexible as WeChat and lacked the full emotional expression made possible with other platforms.

Usually Yolanda's mother initiated the conversation in the family chat group, but she preferred talking to typing so rarely sent text messages, instead she favored the playful use of stickers. Yolanda told her parents that she still had a scholarship for the semester and her mother replied with a sticker saying, "So Happy." Yolanda then registered her own official account on WeChat and started writing fashion blogs on her public account. 


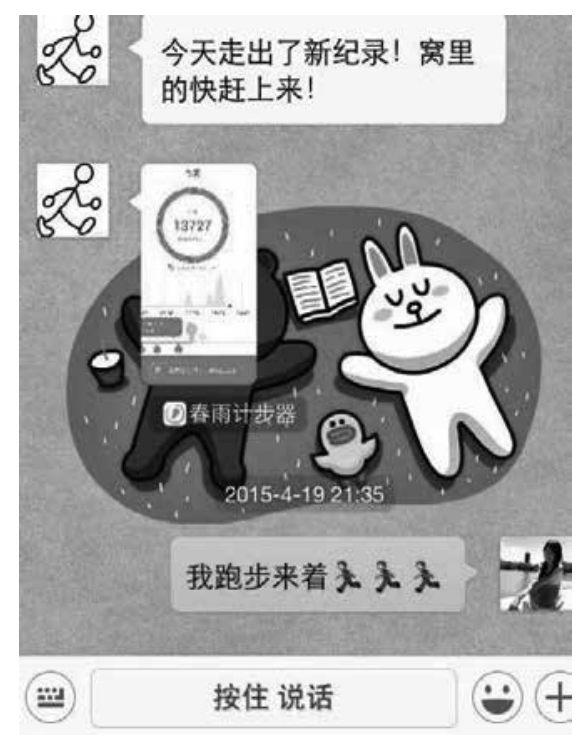

Figure 2.2: Yolanda's father sharing his daily jogging record in the family WeChat Group

Her parents subscribed to her official account to read her blogs and invited their friends to follow, which made Yolanda feel a little awkward in that she did not regard her parents' friends as her target audience. But she still appreciated her parents' passionate support.

Yolanda taught her parents how to use WeChat during the times she visited home, such as during the Spring Festival. Her mother asked about the WeChat Red Packet and so Yolanda spent time figuring out how it worked. She liked to encourage her parents to use digital media to help them discover and cultivate their interests. Considering that her parents planned to retire in 10 years, she wanted them to gain experience with digital media for their retirement. Yolanda also encouraged her parents to share in Moments of WeChat. Due to their concern for privacy, initially Yolanda's parents told her not to share too much on social media. She hoped that her parents would share more in Moments so that the family could learn more about each other by reading each other's social media posts.

\section{Melbourne: Facebook, WhatsApp and Instagram}

Unlike Shanghai and Tokyo where one platform dominates social media, social mobile media practices in Melbourne reflect what Madianou and 


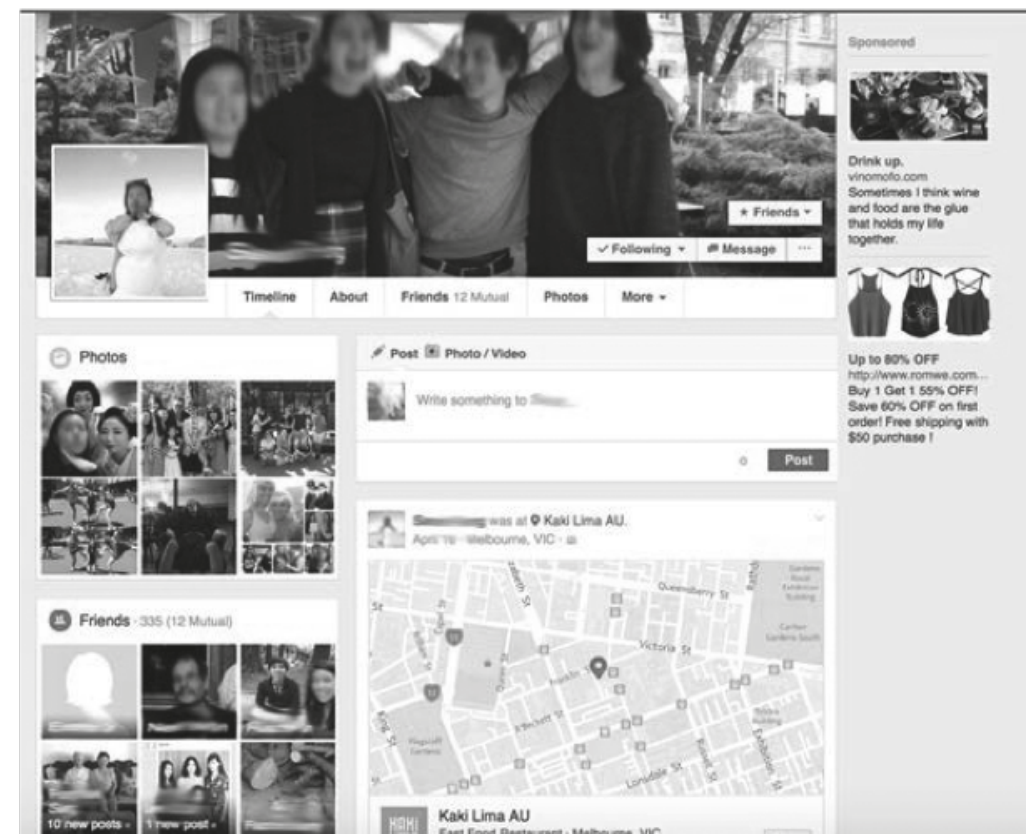

Figure 2.3: Facebook page from our Melbourne participants

Miller (2013) call "polymedia." The concept of polymedia attempts to understand the social and cultural dimensions around the use of increasingly diverse platform technologies as part of the ever-changing interpersonal communication channels. In Melbourne this includes a mix of Facebook, WhatsApp and Instagram.

According to Social Media News (2016), Facebook has 15 million Australian users. Facebook emerged as the dominant social media platform between 2007 and 2008, overtaking then popular social networking sites such as MySpace. The most prominent feature of Facebook, the "profile," operates in a similar way to a bulletin board, where users post textual updates (or "status"), images, videos, links and curated "life events"; all of which create the "timeline" or "newsfeed." Once logging on or checking Facebook from a smartphone, users see posts by other "friends" in their networks appear chronologically in their timeline.

Interaction with posts by others encourages leaving a "like" in the form of paralinguistics — clicking on a thumbs-up icon or another emotional reaction e.g. wow, sad-or by leaving a comment that can instigate a conversation. Members can also create groups, which allows those with mutual interests to share, update and discuss. They also have the ability to create events to inform, publicize, invite guests, and keep track of 
attendance numbers; to manage pages around a particular interest or business; and to private message friends within a confined space. The growth of interaction from once just a "like" to now six different reactions reflects a broader trend in the increasing personalization and affective labor in social media practices. Facebook and the linked Facebook Messenger app also have its own version of stickers. Here we can see the platform proprietary of stickers as much like a type of vernacular or local platform paralinguistics.

Wilken (2014) has also noted the growing importance of Facebook as a location-based platform since it increased locative capacities in 2010; to allow Facebook to dominate as a location-based services company, a recommendation service and to allow for more, targeted advertising campaigns. Yet, as we explore further in this book, the concerns for location, data, privacy and surveillance vary from friendly surveillance as location as known by others in one's networks and data privacy in relation to third parties, companies and government bodies.

WhatsApp has far fewer Australian users-approximately 2.4 million-and our study suggests that WhatsApp is more popular with individuals who have friends and relatives overseas. In our study this was most prominent among migrant families with connections in Southeast Asia. WhatsApp gained popularity in relation to its predecessor, BlackBerry Messenger (BBM), which could only be used between BlackBerry users. When Samsung and other Android phones became prominent competitors to the BlackBerry and iPhone markets, WhatsApp gained traction as it could be used across infrastructural platforms, between users of different smartphones.

Similar to BBM, WhatsApp replicates mobile phone text messaging functions, but uses broadband WiFi to enable unlimited "free" message exchange, phone calls and video calls. Messages accumulate in the form of a chat between two or more individuals as a group. The group chat function has emerged as an important feature throughout the fieldwork in Melbourne, for those who belong to vocational groups and for families with relatives overseas. Within messages, people can also send links, video and audio clips, and images. Image exchange has become an important way of facilitating ambient co-presence and sharing mundane aspects of everyday life as a form of bonding. Messages include a time stamp and one tick appears when the messages have been sent, two ticks appear when the message has been received and turn blue when the message has been read. Individuals can also see the last time their correspondent was online, if they are currently online, and when they are typing a message. 


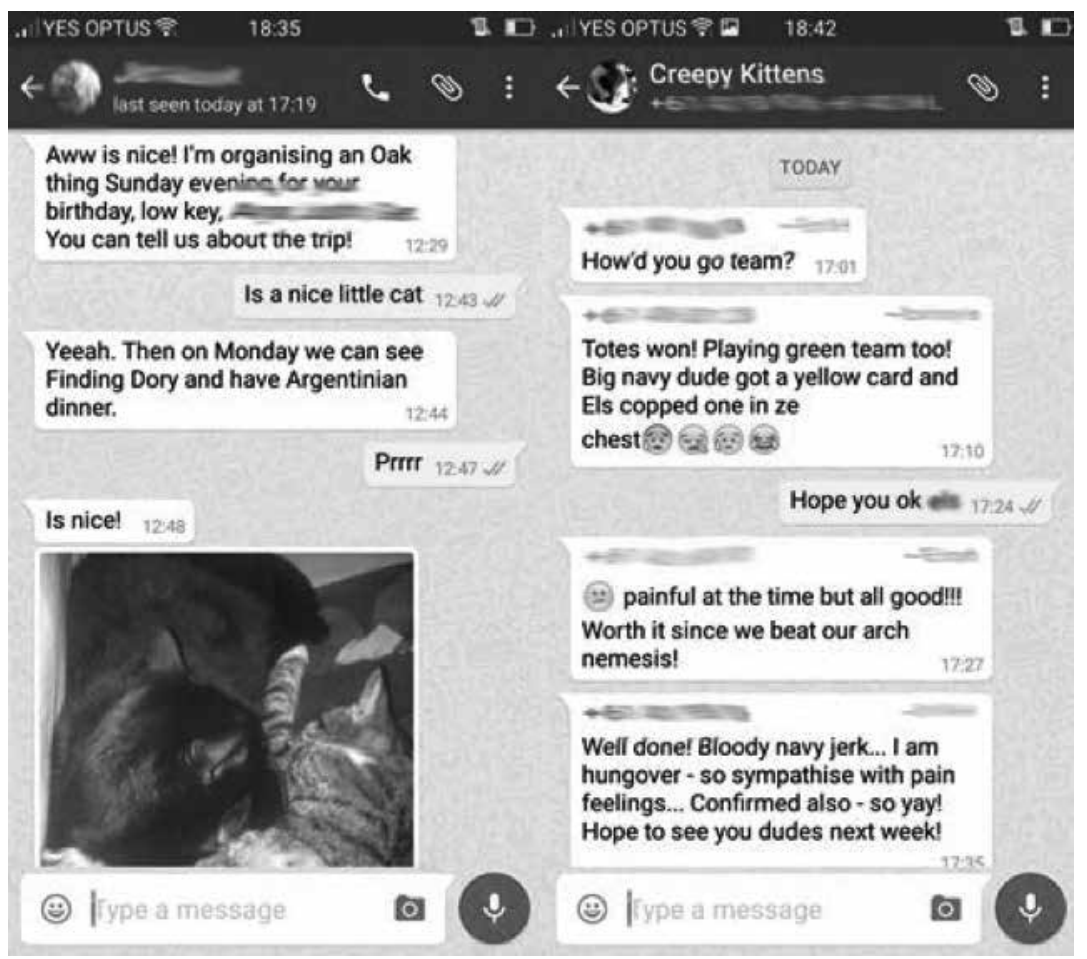

Figure 2.4: WhatsApp

WhatsApp currently does not display location data between those communicating although location data is collected through the platform as accessed on a smartphone. One of the main uses of WhatsApp is the use of groups. This function challenges popular arguments that social media facilitates ego-centered networking. Throughout our fieldwork in Melbourne, group chats were used in a variety of ways. Take 25 -year-old Jasmine as an example. She joined a family group chat, consisting mostly of relatives overseas after a cousin had a baby. The cousin posted images of her newborn every day and different relatives would comment, often resulting in an ongoing conversation. Jasmine herself did not respond often to the chat messages but liked being included as a "lurker" who could read and listen to the messages. Other participants were far more active within group chats.

30-year-old Lily was involved in two WhatsApp groups, one with her close friends from church and the other a mothers' group. The mothers' group exchanged recipes and photos of dishes they were preparing at least a few days a week. When we visited Lily, it was obvious her passions were cooking and baking. She had a bookshelf of cookbooks, exercise books with 
handwritten recipes, and recipes she had printed out from the internet. She also had a food app on her phone that allowed her to browse and share recipes. The WhatsApp group was yet another way for Lily to collect, store and share recipes.

WhatsApp groups were also used between extended family members living overseas for coordinating vocational interest groups. Daniel, for example, was in his late 5os and an active member of a men's choir. They had a WhatsApp group chat to update each other of rehearsal schedules and upcoming events, and exchanged news links and some banter through the week between seeing each other.

Similarly, 6o-year-old Esther and 57-year-old Stephen were a brother and sister who moved to Australia with their families. Since joining Facebook and WhatsApp, they had become far more active within group conversations, mostly on WhatsApp. Esther socialized in a group with her old high school friends from the 1970s, which had over 60 members. Esther received several picture messages every few weeks. She noted that she routinely deleted images that involved politics or anything crude. She kept old photos that her friends scanned and sent, memes of greetings or with sayings she found positive or motivational, and holiday photos. She manually backed up images sent over WhatsApp from her phone to her laptop every few months. Esther found that rekindling a relationship with her old friends from Malaysia was a pleasant and relaxing distraction at the end of her workday.

Like Esther who socialized in WhatsApp with old friends, Stephen participated in a WhatsApp group with his ex-navy colleagues where they exchanged family and travel photos. They also sent funny memes or images that Stephen kept on his phone and looked at from time to time. Stephen also administered his ex-navy colleagues' Facebook page, but he found that the group posted minimally on the page because the WhatsApp group chat was so active. Family in these WhatsApp groups were the main content that members shared.

\section{Locating the Platforms Across the Sites: Emojis/Stamps/Stickers}

In each of the locations, uptake of specific platforms operates in relation to familial relationships. Participants shaped the platform to perform particular forms of affect and co-present intimacy. Across all the different platforms discussed, emojis/stamps/stickers played an important role in everyday mundane rituals - especially when physically co-located. Here we see the playful characteristic of contemporary media come to the forefront (Sicart 
2014). The use of emoticons - emoji-has become an increasingly popular and playful way of personalizing digital media communications.

As one Japanese participant 34-year-old Mana noted, she shared lots of stamps to send love to geographically distant family members. For Mana, a great deal of time was spent using creatively situational stamps, such as "Cute!" and "Take care!" to create a sense of participation and being there. The stamps gave a sense of feeling and emotional that can be hard to express in words.

As discussed earlier, in Asian countries such as Japan, the historical trajectories towards emoji use are tied to the role of the kawaii. The historical context in Japan offers a starting point for considering how such practices have come about and suggests that we would likewise find similar but differently visible forms of cute personalization in other cultures. We have found that with the now ubiquitous (albeit uneven) uptake of mobile social media in the Asia-Pacific region, localized forms of cute character culture have become prevalent across cultures and intergenerationally. Our research has shown how grandparents, parents and children across our Australian, Japanese and Chinese research sites, can all be found deploying different forms of paralinguistics-emojis, emoticons and social media stickers (i.e. LINE, WeChat, WhatsApp) —in playful ways that are tied to generational norms, as well as providing new ways to transgress intergenerational boundaries. We argue that paralinguistics like emoji and stickers can provide an affective care at a distance and co-present intimacy that not only helps to bind families when they are experiencing geographic distance but also provides playful and creative ways for intergenerational literacy and lasting connection.

Through the three different case studies described, we can see the role of paralinguistics in sustaining intimacy intergenerationally-especially when distance is an issue. As we saw in the case of Japan and China, young adults teach their parents to use paralinguistic methods when they move away from home as a way to keep constant contact. In Japan and Australia, the usage between mothers and daughters dominate. Whereas in Australia, it is predominantly migrant participants who use paralinguistics the mostespecially for keeping in close contact with young family members abroad.

Paralinguistics, we suggest, provide a space to explore emotions in ways that traditional forms of language in media platforms cannot. Entangled within everyday mundane digital media practices of care (see McKay 2007; Yeates 2004; Lynch and McLaughlin 1995), our insights into the uses of and feelings that are generated through paralinguistics build on research around family members "do family" at a distance through everyday activities that 
extend beyond the home including through digital media (Morgan 1996; Wajcman et al. 2008; Madianou 2016).

Recent literature in fields such as social media have started to take emojis seriously, as part of the affective labor landscape and playbour of contemporary digital media (Stark and Crawford 2015). Work and young people have been the primary focus, leaving little research exploring how these affective practices play out within families, especially in terms of families grappling with physical distance. As we see in these three localities, the power of emojis have what Stark and Crawford $(2015,1)$ describe as "conduits for affective labor in the social networks of informational capitalism" become central. Different generations, cultures and labor settings are all deploying the emoji with numerous affective and creative practices. As Stark and Crawford note, emoji help to creatively manage "everyday biopolitics":

The patterns of use for emoji over time between friends and partners can become abstract and cryptic, or can degenerate to become pro forma-just plain basic. In the best case, there is a unique personal subtext to that exchange of a rainbow or the love-heart smile, many layers of unspoken meaning that would be difficult for intelligence analysts or machinelearning algorithm to parse. Nonetheless, this complexity has not stopped institutions from making the attempt, and commercializing emoji sociality in other ways $(2015,6)$.

Paralinguistics, we argue, can be seen as an extension of what Julian Kücklich (2005) called "playbour" - that is, involving emotional, creative and social labor in and around their playfulness. In turn, paralinguistics identify a fundamental paradox underscoring digital labor - that is, it is both a playground and a factory (Scholz 2012). Comparing their use in Shanghai, Tokyo and Melbourne with families, we found some of the vernaculars around paralinguistics including "stamps" help with managing intimacies at a distance. In our research with families where adult children lived away from their parents, paralinguistics and stickers were viewed as an important part of the emotions at a distance, by creating a type of affective warmth that sometimes over exceeded face-to-face contact and intimacy.

We argue that paralinguistics like emoji and stickers can provide an affective care at a distance (continuity) and co-present intimacy that contributes in playful and creative ways to intergenerational literacy and connection. As extensions of historical tropes like the kawaii, emojis help to provide emotional warmth around "cool" new media. This chapter has taken the first steps towards understanding this phenomenon. 
We end by proposing that since paralinguistics appear to be here to stay, at least for the immediate future, and the role that they play in everyday mundane and affective lives needs further ethnographic investigation. This is important since such a research agenda seeks to reveal not simply how and why paralinguistics are important in the affective lives of families, but also suggests how such intergenerational relationships are bound up in the problematic global political economies of digital media, technology and labor that are inseparable from this context. In the next chapter we explore the role of locative media, as an integral part of social mobile media, in recalibrating forms of what we call "friendly surveillance." Like the emotional labor of emojis/stickers/stamps, the use and non-use of locative media provides families with ways in which to make and unmake boundaries of intimacy and kinship.

\section{References}

Bogost, Ian, and Nick Montfort. 2009. "Platform Studies." www.platformstudies. com/. Accessed 21 May 2015.

Byford Sam. 2013. "Path moves toward monetization with stickers and private messaging in 3.o." The Verge. www.theverge.com/2013/3/6/4073002/path-3-0update-available-with-messaging-and-shop. Accessed 21 May 2015.

Foucault, Michel. 2003. The essential Foucault: Selections from essential works of Foucault, 1954-1984. New York: The New Press.

Fujimoto, Kenichi. 2005. “The third stage paradigm.” Personal, portable, pedestrian: Mobile phones in Japanese life, edited by Mizuko Ito, Misa Matsuda, and Daisuke Okabe. 77-102. Cambridge: The MIT Press.

Gill, Tom, Brigitte Steger, and David H. Slater. 2013. Japan copes with calamity. New York: Peter Lang.

Gillespie, Tarleton. 2010. "The Politics of 'Platforms'." New Media \& Society 12: 347-364. DOI: 10.1177/1461444809342738.

Hjorth, Larissa. 2003a. "Kawaii@keitai." Japanese Cybercultures, edited by Nanette Gottlieb and Mark McLelland, 50-59. New York: Routledge.

—. 2003b. "Pop and Ma." Mobile Cultures, edited by Fran Martin, Audrey Yue, and Chris Berry, 158-179. Durham: Duke University Press.

- 2005. "Odours of mobility." Journal of Intercultural Studies 26 (1-2): 39-55.

- 2009. Mobile Media in Asia-Pacific. London: Routledge.

Hjorth, Larissa, and Kyoung-hwa Y. Kim. 2011. "The mourning after: A commentary on crisis management in Japan post 3.11." Television \& New Media Journal 12 (6): 552-559. DOI: 10.1177/1527476411418351. 
Hjorth, Larissa, Sarah Pink, Kristen Sharp, and Linda Williams. 2016. Screen Ecologies. Cambridge:The MIT Press.

Ingold, Tim. 2010. "Bringing things back to life: Creative entanglements in a world of materials." NCRM Working Paper. Realities / Morgan Center, University of Manchester (Unpublished). http://eprints.ncrm.ac.uk/1306/. Accessed 21 May 2015. ITmedia Business Online. 2013. "LINE, Naver matome ha naze tsuyoinoka? (Why LINE and Naver are strong?).” http://bizmakoto.jp/makoto/articles/1309/o4/ newso16_2.html. Accessed 4 August 2015.

Ito, Mizuko, Misa Matsuda, and Daisuke Okabe. eds. 2005. Personal, portable, pedestrian: Mobile phones in Japanese Life. Cambridge: The MIT Press.

Ito, Mizuko, and Daisuke Okabe. 2005. "Intimate visual co-presence." Paper presented at Ubicomp, Takanawa Prince Hotel, Tokyo, Japan, 11-14 September.

Kinsella, Sharon. 1995. "Cuties in Japan." In Women, Media and Consumption in Japan, edited by Lisa Skov and Brian Moeran, 220-54. London: Curzon Press.

Kücklich, Julian. 2005. "Modders and the games industry." Fibreculture Journal Issue 5. http://five.fibreculturejournal.org/fcj-025-precarious-playbour-moddersand-the-digital-games-industry/. Accessed 4 August 2015.

LINE. 2015. "2015 nen 4 gatsu - 9 gatsu baitai shiryou (LINE media guide 2015 AprSep)." https://linecorp.com/ads/pdf/8CCCEF52-B730-11E4-BEB8-ED3 AF5 F15 F22. Accessed 4 August 2015.

Madianou, Mirca, and Daniel Miller. 2013. "Polymedia: Towards a new theory of digital media in interpersonal communication." International Journal of Cultural Studies 16 (2): 169-187. DOI: 10.1177/1367877912452486.

Scholz, Trebor. Ed. 2012. Digital labour: The internet as playground and factory. New York: Routledge.

Sicart, Miguel. 2014. Play Matters. Cambridge: The MIT Press.

Slater, David H., Keiko Nishimura, and Love Kindstrand. 2012. "Social media, information and political activism in Japan's 3.11 crisis." The Asia-Pacific Journal, Japan Focus 10 (24):1-33. https://apjjf.org/2012/10/24/David-H.-Slater/3762/article. html. Accessed 21 May 2015.

Social Media News. 2016. "Social Media Statistics - August 2015." http://www. socialmedianews.com.au/social-media-statistics-australia-august- 2015/. Accessed 21 May 205.

Stark, Luke, and Kate Crawford. 2015. "The conservatism of emoji: Work, affect, and communication." Social Media \& Society July-December.1-11. DOI: $10.1177 / 2056305115604853$.

Tencent. 2016. “2016 Annual Report.” http://www.tencent.com/zh-cn/content/ir/ rp/2016/attachments/201601.pdf. (In Chinese). Accessed 2 May 2017.

van Dijck, José. 2016. "Public Values in a Platform Society.” AoiR keynote, Berlin October 2016. 
Weather News. 2011. "A survey on "stranded commuters." Tokyo Metropolitan area on the day of Great East Japan Earthquake, 11 March 2011. http://weathernews. com/ja/nc/press/2011/110411_2.html. Accessed 31 October 2015.

Wilken, Rowan. 2014. "Proximity and Alienation." The Mobile Story: Narrative Practices with Locative Technologies, edited by Jason Farman, 175-191. New York: Routledge. 
